\title{
Electro-weak production of pseudovector C-even heavy quarkonia in electron-positron collisions on Belle II and BES III
}

\author{
Nikolay Achasov ${ }^{1, *}$ \\ ${ }^{1}$ Sobolev Institute for Mathematics, 630090 Novosibirsk, Russia
}

\author{
Abstract. \\ 1. The $X(3872)$ State as Charmonium $\chi_{c 1}(2 P)$ \\ 2. The $\chi_{c 1}$ and $\chi_{b 1}$ production in the $e^{+} e^{-} \rightarrow \chi_{c 1} / \chi_{b 1}$ reaction
}

\section{OUTLINE}

The $X(3872)=\chi_{c 1}(3872)$ meson [1], a patriarch of the $X Y Z$ spectroscopy, was appointed to be the $D^{0} \bar{D}^{* 0}+$ c.c. molecule with a radius greater than 3 fermi from the very beginning despite the fact that $X(3872)=\chi_{c 1}(3872)$ is produced in hard processes with a radius less than one fermi as intensively as the compact charmonium $\psi(2 S)$. Even the landmark result of the LHCb Collaboration [2]

$$
\frac{B R(X \rightarrow \gamma \psi(2 S))}{B R(X \rightarrow \gamma J / \psi)}=2.46 \pm 0.7,
$$

directly pointing to the charmonium nature of $X(3872)$, did not stop the molecular lobby.

We reviewed the scenario in detail where $X(3872)$ resonance is the $c \bar{c}=\chi_{c 1}(2 P)$ charmonium which "sits on" the $D^{0} \bar{D}^{* 0}$ threshold. We explained all known data on $X(3872)$ and suggested clear program of verification of our scenario [3-5].

We predicted the significant number of decay channels via two gluons: $X(3872) \rightarrow$ gluongluon $\rightarrow$ lighthadrons, the same as in the case $\chi_{c 1}(1 P) \rightarrow$ gluongluon $\rightarrow$ light hadrons. It means that two virtual gluons can produced the $X(3872)$ resonance

$$
e^{+} e^{-} \rightarrow \psi\left(m_{i}\right) \rightarrow \gamma \text { gluon gluon } \rightarrow \gamma X(3872),
$$

here $\psi\left(m_{k}\right): I^{G}\left(J^{P C}\right)=0^{-}\left(1^{--}\right), m_{i}>m_{X(3872)}$. The BES III Collaboration found the $X(3872)$ resonance in the reaction $e^{+} e^{-} \rightarrow \gamma X(3872)$ at center-of-mass energies for 4.009 to 4.420 $\mathrm{GeV}[6]$

$$
\begin{aligned}
& e^{+} e^{-} \rightarrow \sum_{i} \psi\left(m_{i}\right) \rightarrow \gamma \text { gluon gluon } \rightarrow \gamma X(3872)= \\
& =\psi(4040)+\psi(4160)+\psi(4230)+\psi(4260)+\psi(4360)+ \\
& +\psi(4390)+\psi(4415) \rightarrow \gamma \text { gluon gluon } \rightarrow \gamma X(3872) \rightarrow \\
& \rightarrow \gamma \pi^{+} \pi^{-} J / \psi
\end{aligned}
$$

\footnotetext{
*e-mail: achasov@math.nsc.ru
} 


\section{$\mathrm{e}^{+} \mathrm{e}^{-} \rightarrow \gamma \pi^{+} \pi^{-} \mathrm{J} / \psi$}

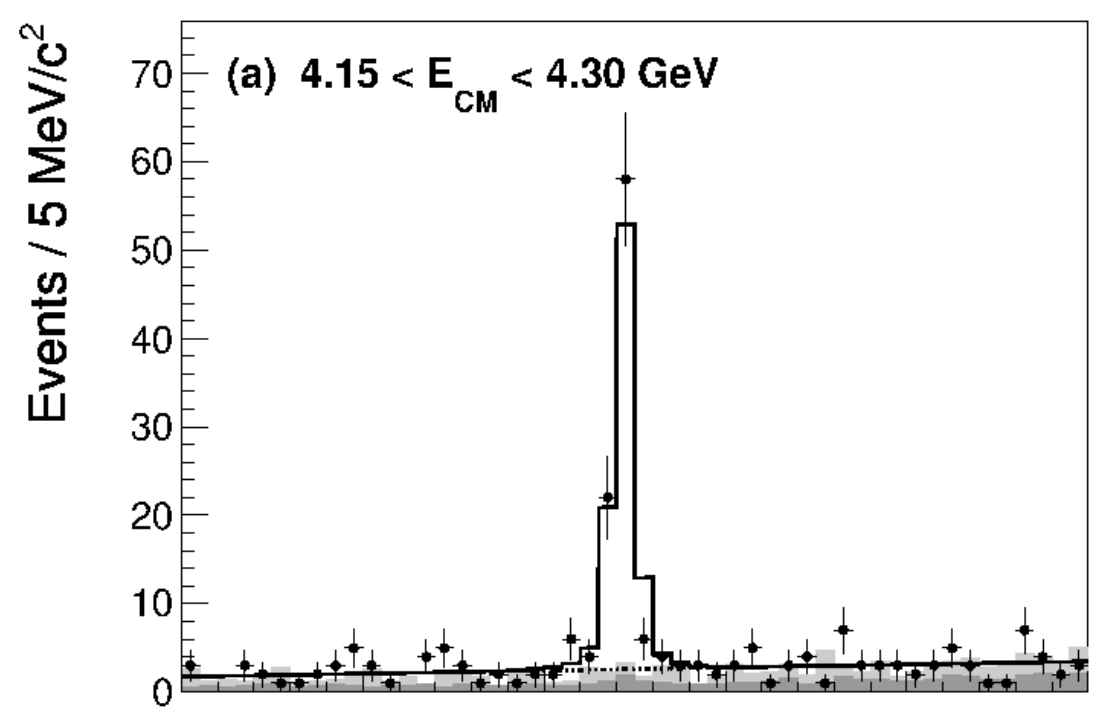

Figure 1. Distribution of $\pi^{+} \pi^{-} J / \psi$ mass from Ref. [7].

Recently the BES III Collaboration found the $X(3872)$ resonance in the reaction $e^{+} e^{-} \rightarrow$ $\gamma X(3872)$ at center-of-mass energies for 4.15 to $4.3 \mathrm{GeV}$ [7]

$$
\begin{aligned}
& e^{+} e^{-} \rightarrow \psi(4160)+\psi(4230)+\psi(4260) \rightarrow \\
& \rightarrow \gamma \text { gluon gluon } \rightarrow \gamma X(3872) \rightarrow \gamma \pi^{+} \pi^{-} J / \psi,
\end{aligned}
$$

see Fig. 1 and

$$
\begin{aligned}
& e^{+} e^{-} \rightarrow \psi(4160)+\psi(4230)+\psi(4260) \rightarrow \\
& \rightarrow \gamma \text { gluon gluon } \rightarrow \gamma X(3872) \rightarrow \gamma \pi^{0} \chi_{c 1}(1 P),
\end{aligned}
$$

see Fig. 2.

The giant colourless molecule does not connected with gluons! Its colourless constituents $D^{0}, \bar{D}^{* 0}$ do not connected with gluons also! So The BES III Collaboration closes the molecular model of the $X(3872)$ resonance.

As for the tetraquark model, the two-gluon production of the $X(3872)$ resonance is possible $e^{+} e^{-} \rightarrow \gamma$ gluon gluon $\rightarrow \gamma q \bar{q} c \bar{c} \rightarrow \gamma X(3872), q=u, d$. But, such a process is described by nonplanar diagrams, which are depressed always. So the BES III collaboration puts in a difficult position the tetraquark model of the $X(3872)$ resonance.

Thus the BES III collaboration confirms the $c \bar{c}$ charmonium model of the $X(3872)$ resonance.

$$
X(3872)=\chi_{c 1}(2 P) !
$$

It is often thought that violations of isotopic invariance in the decays $X(3872) \rightarrow \pi^{+} \pi^{-} J / \psi$ and $X(3872) \rightarrow \pi^{0} \chi_{c 1}(1 P)$ are crucial for the $X(3872)$ nature. 


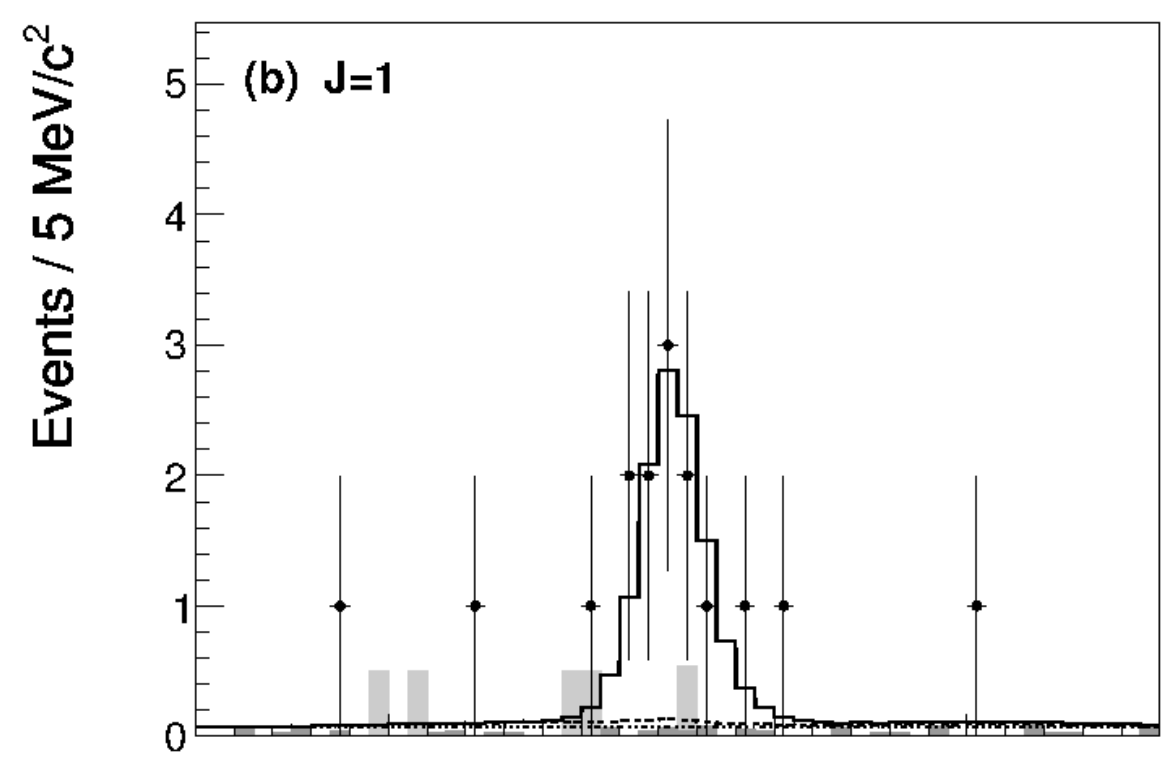

Figure 2. Distribution of $\pi^{0} \chi_{c 1}(1 P)$ mass from [7].

However, this is a misunderstanding. These are the problems of the second row.

The point is that electromagnetic interaction is not small in this energy region, $\alpha \sim \alpha_{s}^{3}$. As a result $B R(J / p s i \rightarrow g g g)=(64.1 \pm 1.0) \%, B R(J / p s i \rightarrow \gamma g g)=(8.8 \pm 1.1) \%, B R(J / p s i \rightarrow$ virtual $\gamma \rightarrow$ hadrons $)=(13.5 \pm 0.30) \%$ [1]. Close to our scenario is an example of the $J / \psi \rightarrow \rho \eta^{\prime}$ and $J / \psi \rightarrow \omega \eta^{\prime}$ decays. According to Ref. [1]

$$
B R\left(J / \psi \rightarrow \rho \eta^{\prime}\right)=(1.05 \pm 0.18) \cdot 10^{-4} \text { and } B R\left(J / \psi \rightarrow \omega \eta^{\prime}\right)=(1.82 \pm 0.21) \cdot 10^{-4}
$$

The similar picture is shown also by the $\psi(2 S)[1]$.

As for the isotopic symmetry violation via $m_{d}-m_{u}$, it can be considerable also, for example, the $\rho^{0}-\omega$ and $\eta-\pi^{0}$ transitions are of the order $\left(m_{d}-m_{u}\right) \times 1 \mathrm{GeV}$ order [8].

As for $X(3872) \rightarrow \pi^{+} \pi^{-} J / \psi$ and $X(3872) \rightarrow \omega J / \psi$, this problem is discussed in detail in Refs. $[4,5]^{1}$. As for $X(3872) \rightarrow \pi^{0} \chi_{c 1}(1 P)$, it is possible a such scheme $X(3872) \rightarrow$ gluon gluon $\chi_{c 1}(1 P) \rightarrow \eta \chi_{c 1}(1 P) \rightarrow \pi^{0} \chi_{c 1}(1 P)$ via $\eta-\pi^{0}$ mixing.

I dare recommend looking for the decays $\chi_{b 1}(2 P) \rightarrow \rho^{0} J / \psi$ and $\chi_{b 1}(2 P) \rightarrow \pi^{0} \chi_{b 1}(1 P)$.

\section{OUTLOOK}

In this energy region the weak interaction grows with energy increase $\propto G_{F} E^{2}$, here $G_{F}=10^{-5} m_{p}^{-2}$ is the Fermi constant.

\footnotetext{
${ }^{1}$ It is interesting to note that else in Ref. [9] there was shown that the $\omega$ do not produced virtually in the $X(3872) \rightarrow \omega J / \psi$ decay. One can see only the left tail of $\omega$ far off the resonance. Let us add that a background can interfere with this tail constructively or destructively. But the molecular lobby hard discusses the strong isotopic breaking in the above decays.
} 


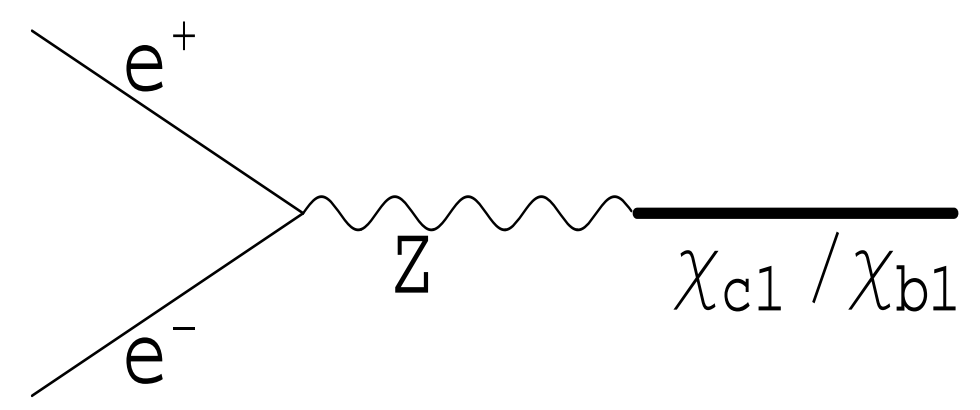

Figure 3. Diagram of the process $e^{+} e^{-} \rightarrow Z \rightarrow \chi_{c 1} / \chi_{b 1}$.

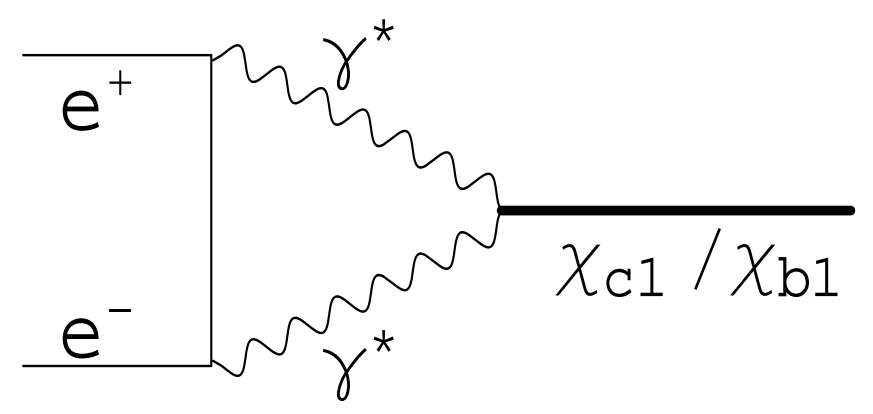

Figure 4. Diagram of the process $e^{+} e^{-} \rightarrow \gamma^{*} \gamma^{*} \rightarrow \chi_{c 1} / \chi_{b 1}$

$G_{F} E^{2}=1.4 \times 10^{-4}$ for $\chi_{c 1}(1 P)$ and $G_{F} E^{2}=1.7 \times 10^{-4}$ for $\chi_{c 1}(3872)$. That is, $G_{F} E^{2} \sim \alpha^{2}$ in the BES III energy region.

$G_{F} E^{2}=1.1 \times 10^{-3}$ for $\chi_{b 1}(1 P)$ and $G_{F} E^{2}=1.2 \times 10^{-3}$ for $\chi_{b 1}(2 P)$. That is, $G_{F} E^{2} \gg \alpha^{2}$ in the Belle II energy region.

The BESS III luminosity $10^{33} \mathrm{~cm}^{-2} \mathrm{~s}^{-1}$ gives possibilities to register near hundred of events of the $e^{+} e^{-} \rightarrow Z \rightarrow \chi_{c 1}(1 P)$ decays, Fig. 3, per day [10] and near thirty of them in the well-known channel $\chi_{c 1}(1 P) \rightarrow \gamma \psi(1 S)$. If $\chi_{c 1}(3872)=\chi_{c 1}(2 P)$, then also near hundred of events of the $e^{+} e^{-} \rightarrow Z \rightarrow \chi_{c 1}$ (3872) decays, Fig. 3, per day may be registered and several of them in the channel $\chi_{c 1}(3872) \rightarrow \gamma \psi(2 S)$, several tens of them in the channel $\chi_{c 1}(3872) \rightarrow D^{0} \bar{D}^{* 0}+$ c.c..

The huge Belle II luminosity $8 \times 10^{35} \mathrm{~cm}^{-2} \mathrm{~s}^{-1}$ gives possibilities to register near hundred thousand of events the each $e^{+} e^{-} \rightarrow Z \rightarrow \chi_{b 1}(1 P)$ and $e^{+} e^{-} \rightarrow Z \rightarrow \chi_{b 1}(2 P)$ decays, Fig. 3, per day [10] and several tens of thousands of them in the well-known channels $\chi_{b 1}(1 P) \rightarrow \gamma^{\Upsilon} \Upsilon(1 S)$ and $\chi_{b 1}(2 P) \rightarrow \gamma^{\Upsilon}(2 S)$.

Note that the above estimations were became under the assumption that the resonance widths are not small compared to the energy resolution. 
In the Belle II energy region will dominate the one-Z-boson mechanism, Fig. 3. As for BESS III energy region, the one-Z-boson mechanism, Fig. 3, and the two-photon mechanism, Fig. 4, are probably of the same order. Fortunately, the $e^{+} e^{-} \rightarrow Z \rightarrow \chi_{c 1} / \chi_{b 1}$ and $e^{+} e^{-} \rightarrow$ $\gamma^{*} \gamma^{*} \rightarrow \chi_{c 1} / \chi_{b 1}$ contributions $^{2}$ do not interfere in the total cross sections. The creation of longitudinally polarized electron-positron beams allows to study both the total cross sections and the interference of $e^{+} e^{-} \rightarrow Z \rightarrow \chi_{c 1} / \chi_{b 1}$ with $e^{+} e^{-} \rightarrow \gamma^{*} \gamma^{*} \rightarrow \chi_{c 1} / \chi_{b 1}$. More specific

$$
\begin{aligned}
& e^{+}(1) e^{-}(-1) \rightarrow \chi_{c 1} / \chi_{b 1}(-1)=e^{+}(1) e^{-}(-1) \rightarrow Z \rightarrow \chi_{c 1} / \chi_{b 1}(-1)+ \\
& +e^{+}(1) e^{-}(-1) \rightarrow \gamma^{*} \gamma^{*} \rightarrow \chi_{c 1} / \chi_{b 1}(-1), \\
& e^{+}(-1) e^{-}(1) \rightarrow \chi_{c 1} / \chi_{b 1}(1)=-(!) e^{+}(-1) e^{-}(+1) \rightarrow Z \rightarrow \chi_{c 1} / \chi_{b 1}(1)+ \\
& +e^{+}(-1) e^{-}(1) \rightarrow \gamma^{*} \gamma^{*} \rightarrow \chi_{c 1} / \chi_{b 1}(1),
\end{aligned}
$$

where $\lambda$ in $e^{+}(\lambda), e^{-}(\lambda)$, and $\chi_{c 1} / \chi_{b 1}(\lambda)$ is the projection of the particle spin on the electron momentum direction in the mass center system. We neglected $m_{e}$.

\section{SUMMARY}

The new elegant experimental probes appear. In particular, they could find out whether is $\chi_{c 1}(3872)=\chi_{c 1}(2 P)$ and search out the $\chi_{b 1}(2 P) \rightarrow \rho^{0} \Upsilon(1 S)$ and $\chi_{b 1}(2 P) \rightarrow \pi^{0} \chi_{b 1}(1 P)$.

\section{Acknowledgments}

I am grateful to Organizers of From Phi to Psi - 2019 for the kind Invitation.

The work was supported by the program No. II.15.1 of fundamental scientific researches of the Siberian Branch of Russian Academy of Sciences, the project No. 0314-2019-0021.

\section{References}

[1] M. Tanabashi et al. (Particle Data Group), Phys. Rev. D 98, 030001 (2018).

[2] R. Aaij, et al. ( LHCb Collaboration), Nucl. Phys. B 886, 665 (2014).

[3] N.N. Achasov and E.V. Rogozina, JETP Lett. 100, 227 (2014).

[4] N.N. Achasov and E.V. Rogozina, Mod. Phys. Lett. A 30, 1550181 (2015);

J.Univ.Sci.Tech.China 46, 574 (2016).

[5] Nikolay Achasov, EPJ Web Conf. 125, 04002 (2016);

N.N. Achasov, Phys. Part. Nucl. 48, 839 (2017);

Nikolay Achasov, EPJ Web Conf. 191, 04002 (2018) .

[6] M. Ablikim et al. (BESIII Collaboration), Phys. Rev. Lett. 112, 092001(2014).

[7] M. Ablikim et al. (BESIII Collaboration), arXiv: 1901.03992 v1 [hep-ex] 13 Jan 2019.

[8] B.L. Ioffe, Phys. Usp. 441211 (2001); Usp. Fiz. Nauk 1711273 (2001).

[9] Mahiko Suzuki, Phys. Rev. D 72, 114013 (2005).

[10] N.N. Achasov, JETP Lett. 63, 601 (1996);

arXiv:hep-ph/9603262v1; arXiv:hep-ph/9604226v2.

[11] N. N. Achasov, Phys. Lett. B bf 287, 213 (1992);

Sov.Phys. JETP 74, 913 (1992) [ZhETF 101, 1713 (1992)];

JETP Lett. 56, 329 (1992);

JETP 76, 5 (1993) [ZhETF 103, 11 (1993)].

[12] N. N. Achasov, Particle World, Vol. 4, No. 10, pp 10-13 (1994).

\footnotetext{
${ }^{2}$ Note that the $\gamma^{*} \gamma^{*} \chi_{c 1} / \chi_{b 1}$ vertex was considered in Refs. $[11,12]$ in details.
} 